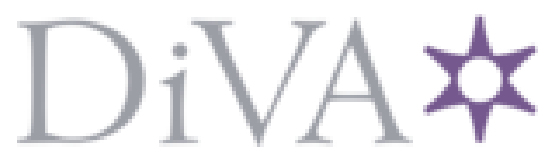

http://www.diva-portal.org

\title{
Preprint
}

This is the submitted version of a paper published in Biochemical and Biophysical Research Communications - BBRC.

Citation for the original published paper (version of record):

Turpaev, K., Welsh, N. (2015)

Brusatol inhibits the response of cultured beta-cells to pro-inflammatory cytokines in vitro.

Biochemical and Biophysical Research Communications - BBRC, 460(3): 868-872

http://dx.doi.org/10.1016/j.bbrc.2015.03.124

Access to the published version may require subscription.

N.B. When citing this work, cite the original published paper.

Permanent link to this version:

http://urn.kb.se/resolve?urn=urn:nbn:se:uu:diva-262449 


\section{Brusatol inhibits the response of cultured beta-cells to pro-inflammatory}

2 cytokines in vitro

3

$4 \quad$ Kyril Turpaev $^{\text {a,b,* }}$, Nils Welsh ${ }^{\text {a }}$

5

$6{ }^{a}$ Department of Medical Cell Biology, Uppsala University, Biomedicum, SE-751 23, Uppsala,

$7 \quad$ Sweden

$8 \quad{ }^{b}$ Center for Theoretical Problems of Physicochemical Pharmacology, Russian Academy of

9 Sciences, 119991 Moscow, Russia

10

11 * Corresponding author:

12 Dr. Kyril Turpaev, Dept. of Medical Cell Biology, Uppsala University, Biomedicum, P.O. Box

13 571, S-751 23, Uppsala, Sweden. Telephone: +46 1847142 12, Telefax: +46 184714059.

14 Permanent address: Center for Theoretical Problems of Physicochemical Pharmacology, Russian

15 Academy of Sciences, 119991 Moscow, Russia.

16 E-mail: kyril.turpaev@yahoo.com

17 


\section{Abstract}

19 Brusatol is a natural terpenoid that is capable of inducing a variety of biological effects. We presently report that this substance dramatically improves beta-cell survival when exposed to pro-inflammatory cytokines (IL-1 $\beta$ and IFN $\gamma$ ) in vitro. This was observed in insulin producing rat (RIN-5AH), mouse ( $\beta$ TC6) and human (EndoC- $\beta$ H1) beta-cell lines. Brusatol prevented betacell oxidative stress in response to cytokines and counteracted induction of iNOS on the protein level. Brusatol, however, block neither the cytokine-induced increase of iNOS mRNA, nor NF$\kappa \mathrm{B}$ activation, suggesting that inhibition of $\mathrm{iNOS}$ protein expression relies on posttranscriptional mechanism. This indicates that brusatol acts via a novel protective pathway, which may represent a more promising way of improving beta-cell function and survival.

Keywords: brusatol; cytokines; beta-cells; oxidative stress; iNOS.

Abbreviations: DCFDA, 2'-7'-dichlorodihydrofluorescein diacetate; iNOS, inducible nitric oxide synthase; PI, propidium iodide; RNS, reactive nitrogen species; ROS, reactive oxygen species. 


\section{Introduction}

Brusatol is a member of quassinoids, group of chemical compounds related to triterpenoids, which can be isolated from Brucea javanica (L.), an evergreen shrub grown in

Southeast Asia. Brusatol and structurally related natural compounds (bruceins, bruceantin, bruceoside, and others) are capable of inducing a variety of biological effects including antimalarial and antineoplastic activities. A major molecular mechanism responsible for its antiplasmoidal activity has been attributed to inhibition of protein synthesis when used in relatively high micromolar concentrations [1]. Brusatol and bruceantin used at a low (about 50 $\mathrm{nM}$ ) concentration were found to induce terminal differentiation and inhibit proliferation of leukemic cells. On the molecular level these effects occurred in parallel with significant protein synthesis and stability. Apparently, the ability of brusatol to reduce the protein level of Nrf2 is unrelated to the Keap1 inhibitory factor, which typically directs Nrf2 to E3 ubiquitin ligase [3].

Pro-inflammatory cytokines as IL-1 $\beta$ and IFN $\gamma$ are produced by activated T cells and macrophages that infiltrate into pancreatic islets under pathophysiological conditions. These cytokines induce a decreased insulin secretion and even beta-cell destruction achieved via concerted actions on several intracellular signaling pathways, including the generation of reactive oxygen (ROS) and nitrogen (RNS) species, MAP kinases, and the NF- $\kappa \mathrm{B}$ transcription factor. Activation of several stress kinases and NF- $\kappa B$ by cytokines and ROS in turn stimulates the expression of pro-inflammatory factors as TNF $\alpha$, IL-6, and monocyte chemoattractant 
protein (MCP-1) which further increases ROS production, thus creating a dangerous feedback loop [4-6]. In the present study we aimed at investigating the putative effects of brusatol on betacell responses to pro-inflammatory cytokines. We observed a remarkable efficacy of brusatol in protecting beta-cells against cytokine-induced oxidative stress and death, and we speculate that this may be explained by its ability to block cytokine-induced signaling without affecting NF- $\mathrm{B}$ transcriptional activity.

\section{Materials and methods}

\subsection{Reagents}

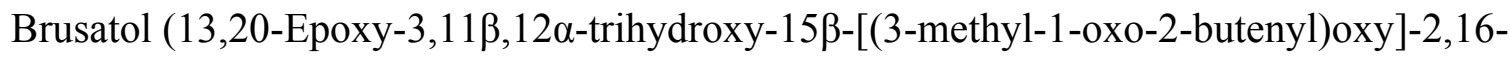
dioxopicras-3-en-21-oic acid methyl ester), and DCFDA (2'-7'-dichlorodihydrofluorescein diacetate) were from Shanghai Tauto Biotech Co (China), and Invitrogen (Carlsbad, CA), respectively. For cell treatments brusatol and DCFDA were dissolved in DMSO. In all experiments with cell cultures, the final DMSO concentration in the medium did not exceed 0.05\%. Propidium iodide (PI) was from Sigma-Aldrich, St. Louis, MO.

\subsection{Cells cultures and nuclear isolation}

Mouse $\beta$ TC6 and rat RIN-5AH cells were grown in RPMI-1640 (Gibco, Grand Island, NY) as described [7]. Human immortalized transgenic EndoC- $\beta \mathrm{H} 1$ cells were grown in serum- 
82 free DMEM medium as described [8]. Cells were cultivated in 24-well plates (Falcon,) to a

83 density about $5 \times 10^{4}$ cells/well. In several experiments cells were treated with cytokines (20

$84 \mathrm{ng} / \mathrm{ml} \mathrm{IL-1} \beta$ (murine) and $20 \mathrm{ng} / \mathrm{ml}$ IFN $\gamma$ (murine or human)) (Peprotech, Rocky Hill, NJ) for a

85

86

87 time as indicated. $\beta$ TC6 and RIN-5AH cells were exposed to murine IFN $\gamma$; EndoC- $\beta \mathrm{H} 1$ cells were exposed to human IFN $\gamma$. All three cell lines were treated with murine IL-1 $\beta$. For nuclear isolation $\beta$ TC6 cells were exposed to IL-1 $\beta$ ( $20 \mathrm{ng} / \mathrm{ml})$ with or without brusatol $(50 \mathrm{nM})$ for the time indicated in the text and subsequently treated as described [2].

\subsection{Analysis of cell viability}

$\beta$ TC6, RIN-5AH or EndoC- $\beta$ H1 cells were cultivated in 24-well plates at the presence or absence of brusatol $(50 \mathrm{nM})$ for 30 min prior to simultaneous exposure to IL-1 $\beta$ and IFN $\gamma$ for additional $24 \mathrm{~h}$. Cell cultures were labeled for $10 \mathrm{~min}$ at $37^{\circ} \mathrm{C}$ with $1 \mu \mathrm{g} / \mathrm{ml}$ of PI. The cells were washed once with PBS and then trypsinized for $5 \mathrm{~min}$ at $37^{\circ} \mathrm{C}$. Cell suspensions were analyzed in a FACSCalibur flow cytometer (Becton Dickinson, Franklin Lakes, NJ) for FL3 fluorescence.

\subsection{ROS generation assay}

\section{well plates at the presence or absence of brusatol $(50 \mathrm{nM})$ for $30 \mathrm{~min}$ prior to exposure to} combination of IL-1 $\beta(10 \mathrm{ng} / \mathrm{ml})$ and IFN $\gamma(10 \mathrm{ng} / \mathrm{ml})$ for $11 \mathrm{~h}$. At the end of incubation period cells were washed with PBS and exposed to DCFDA $(10 \mu \mathrm{M})$ in serum-free RPMI medium for 
$45 \mathrm{~min}$ at $37^{\circ} \mathrm{C}$. The cells were washed with PBS and then trypsinized. Intensity of fluorescence (FL1 channel) was measured by flow cytometry.

\subsection{Western blotting}

8

$\beta$ TC6 cells were washed twice with ice-cold phosphate-buffered saline and whole cell extracts were prepared in SDS sample buffer as described [7]. The following antibodies were

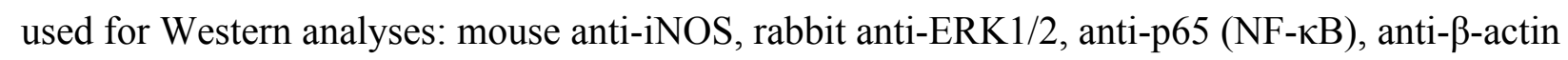
(Santa Cruz Biotechnology, Santa Cruz, CA), and rabbit anti-PTBP2 (Millipore, Billerica, MA). For visualization anti-rabbit and anti-mouse horseradish peroxidase-coupled secondary goat antibodies (Dako, Glostrup, Denmark) were used.

\subsection{RNA extraction and quantitative-real-time PCR analysis}

RNA was purified from $\beta$ TC6 cells using the Qiagen RNeasy kit (Qiagen, Hilden, Germany). cDNA synthesis and quantitative real-time PCR (RT-PCR) were performed as described [7]. For cDNA amplification were used the following primers: iNOS, 5'CCTGGTACGGGCATTGCTCC -3' (forward) and 5'- GCTCATGCGGCCTCCTTTGA -3' (reverse); GAPDH, 5'- AGGTCGGTGTGAACGGATTTG-3' (forward) and 5'TGTAGACCATGTAGTTGAGGTCA-3' (reverse). Values were normalized to the relative amounts of GAPDH cDNA. 


\section{Results}

\subsection{Protective effects of brusatol against cytokine-induced toxicity}

The primary aim of this study was to examine the ability of brusatol to counteract toxic

effects of pro-inflammatory cytokines on pancreatic beta-cells. To that end we analyzed the response on parallel treatment with brusatol and cytokines of three different lines, insulinomaderived murine $\beta$ TC6 and rat RIN-5AH and human genetically transformed EndoC- $\beta \mathrm{H} 1$. Cells were pretreated with brusatol for 30 min before simultaneous exposure to the combination of IFN $\gamma$ and IL-1 $\beta$ for $24 \mathrm{~h}$. After harvest cell viability was assessed by PI staining. As shown in

Fig. 1A, murine $\beta$ TC6 cells were extremely vulnerable to simultaneous treatment with IFN $\gamma$ and IL-1 $\beta$. Cytokines caused an increase of the amount of dead cells up to approximately $90 \%$ of the complete protection of the $\beta$ TC6 cells against inflammatory destruction. Rat RIN-5AH and human EndoC- $\beta \mathrm{H} 1$ pancreatic cells were relatively less sensitive to cytokine-mediated toxicity than murine $\beta$ TC6 cells. Exposure to IFN $\gamma$ and IL- $1 \beta$ for $24 \mathrm{~h}$ led to the death of 55 and $21 \%$ of the total cell populations, respectively. Again, treatment of RIN-5H1 and EndoC- $\beta \mathrm{H} 1$ cells with brusatol resulted in complete suppression of cytokine-induced toxicity (Fig. 1, B and C). Of note, that brusatol did not induce any toxic effect on examined beta-cell lines after $24-\mathrm{h}$ treatment as measured by PI staining. When RIN-5AH and EndoC- $\beta \mathrm{H} 1$ cells were exposed to brusatol alone for $24 \mathrm{~h}$ the basal level of cell death remained unaffected whereas in $\beta$ TC6 brusatol even decreased cell death level compared with untreated control cells (15 and 9\% of the total cell number, respectively) (Fig. 1A). 

cells exposed to IL-1 $\beta$ and IFN $\gamma$. As shown in Fig. 2, the fraction of dead cells decreased dosedependently in $\beta$ TC6 cells in response to brustol concentrations up to $10 \mathrm{nM}$.

It is well established that exposure of beta-cell to pro-inflammatory cytokines stimulates them to increase ROS production through variety pathways such as activation of NADPH oxidases, induction of inducible nitric oxide synthase (iNOS), and alterations in mitochondrial metabolism [5]. In an attempt to understand whether brusatol can attenuate cytokine-mediated oxidative stress we measured intracellular ROS levels in $\beta$ TC6 cells exposed to combination of IFN $\gamma$ and IL-1 $\beta$ with or without prior incubation with brusatol. For ROS detection we used cell permeable fluorescent probe DCFDA. The intensity of DCFDA-derived fluorescence is an indicator of cell levels of hydrogen peroxide, peroxynitrite, nitrogen dioxide as well as hydroxyl, peroxyl, and alkoxyl radicals [9]. As shown in Fig. 3, exposure of $\beta$ TC6 cells to proinflammatory cytokines resulted in an about 2.5-fold increase of DCFDA-based fluorescence. Pretreatment with brusatol resulted in complete suppression of the cytokine-dependent increase of intracellular ROS levels. Moreover, the basal ROS level decreased by about $30 \%$ in cells that were treated with brusatol alone. 
An increase of iNOS level is recognized as principal indicator of beta-cell response on

172 pro-inflammatory cytokines. Moreover, nitric oxide is essential mediator of cytokine-induced

173

174

175 damage of rodent beta-cells. In comparison with other pro-inflammatory cytokines (IFN $\gamma$, TNF $\alpha$ ), in $\beta$ TC6 cells the iNOS expression is most sensitive to IL-1 $\beta$ (our unpublished data). Cytokine-dependent iNOS induction is mediated by the NF- $\kappa \mathrm{B}$ signaling pathway and transcriptional activation of iNOS gene. As shown in Fig. 4A, in unstimulated $\beta$ TC6 cells the iNOS protein level was hardly detectable, but was markedly increased after exposure to IL-1 $\beta$. Combined treatment of $\beta$ TC6 cells with IL-1 $\beta$ and brusatol resulted in significant, about 10 -fold decrease of iNOS protein level (Fig. 4B).

Next we examined the possibility that brusatol reduces the sensitivity of iNOS expression to IL-1 $\beta$ through inhibition of mRNA transcription. RT-PCR analysis showed that exposure of $\beta$ TC6 cells to IL-1 $\beta$ resulted in more than 300-fold increase of iNOS mRNA expression.

Nevertheless, coincubation with brusatol resulted in insignificant attenuation of IL-1 $\beta$-dependent transcriptional activation iNOS gene. An increase of iNOS mRNA level in $\beta$ TC6 cells exposed to IL-1 $\beta$ at the presence of brusatol was attenuated by about $25 \%$ in compare with cells treated with IL-1 $\beta$ alone (Fig. 4B). Accordingly, coincubation of cells with brusatol did not prevent IL$1 \beta$-mediated stimulation of the NF-kB subunit p65 nuclear accumulation whereas exposure of $\beta$ TC6 cells to IL-1 $\beta$ alone resulted in a significant increase of the p65 nuclear level (Fig. 4C). Taken together these findings suggest that brusatol affects the IL-1 $\beta$-dependent increase of iNOS protein levels via posttranscriptional mechanisms. 


\section{Discussion}

In this study we have shown for the first time that brusatol suppresses the sensitivity of pancreatic beta-cells to pro-inflammatory cytokines IL-1 $\beta$ and IFN $\gamma$. We analyzed three typical responses of beta-cells on cytokine challenge: cell death, increase in ROS production, and iNOS induction. Complete rescue from cytokine toxicity was shown for three different cell lines, insulinoma-derived rodent $\beta$ TC6 and RIN-5AH cells and human immortalized EndoC- $\beta \mathrm{H} 1$ cells. On $\beta$ TC6 cells we showed total suppression of cytokine-mediated oxidative stress and significant attenuation IL-1 $\beta$-dependent iNOS protein induction. It is well established that beta-cells are highly vulnerable to pro-inflammatory cytokines. Under pathological conditions these cytokines are produced by activated macrophages and T lymphocytes infiltrated into islets. Proinflammatory cytokines not only play a role in beta-cell destruction but are also implicated in control of lymphocyte maturation and activity. Cytokines directly induce extensive death of betacells through both necrotic and apoptotic mechanisms requiring parallel activation of multiple intracellular signaling pathways $[5,6,10]$.

Apparently, the mechanism through which brusatol attenuates the beta-cell response to pro-inflammatory cytokines is mediated by multiple factors and remains unclear. Cytokinedependent destruction of beta-cells occurs probably in part via activation of NADPH oxidases and induction of iNOS, which will increase the endogenous generation of ROS and RNS. Highly localized concentrations of ROS and RNS can induce oxidative stress leading to multiple cell injuries. In addition, ROS and RNS are acting as second messengers further promoting expression of pro-inflammatory cytokines such as IL-1 $\beta$ and TNF- $\alpha$ in macrophages and dendritic cells $[5,11]$. Islet beta-cells are particularly defenseless against oxidative stress because of low levels of antioxidant enzymes [12]. Thus, approaches aimed to increase beta-cell 
resistance to cytokine-mediated signaling may be effective in prevention of diabetes progression and improving the function and survival of grafted islets.

Here we have shown that brusatol markedly reversed accumulation of the iNOS protein in pancreatic $\beta$ TC6 cells exposed to IL-1 $\beta$. These data are in line with a recent report showing that brusatol and its synthetic derivatives inhibit iNOS induction in murine peritoneal macrophages stimulated with lipopolysaccharides (LPS) [13]. Since brusatol caused only a minor attenuation of the IL-1 $\beta$-dependent increase of iNOS mRNA expression, and did not affect p65 (NF- $\kappa \mathrm{B})$ nuclear accumulation, it is likely that in $\beta$ TC6 cells the inhibitory effect is mediated by unidentified posttranscriptional mechanism, i.e. via translational or post-translational control. The notion is in agreement with a previous report stating that in A549 cells TNF $\alpha$-dependent $\mathrm{NF}-\kappa \mathrm{B}$ activation was insensitive to brusatol when applied at nanomolar concentrations (up to 80 $\mathrm{nM}$ ) [3]. In an earlier study decreased NF- $\kappa \mathrm{B}$ activation was detected in brusatol-treated HL-60 cells when exposed to significantly higher (micromolar) concentrations [2]. An inhibitory effect of brusatol on the transcription factor Nrf2 is well established and has been demonstrated in cell lines such as A549, HT22, Hepa-1c1c7 [3,14,15], and in $\beta$ TC6 (our unpublished data).

Nevertheless, Nrf2 down-regulation is unlikely to mediate the anti-iNOS activity of brusatol since the Keap1/Nrf2 system is not implicated in regulation of iNOS expression. Moreover, brusatol-induced Nrf2 depletion is transient and the basal levels restore within 8-12 $\mathrm{h}$ of exposure $[3,15]$, whereas in our study brusatol protected against cell death induced by IL-1 $\beta$ and IFN $\gamma$ throughout a $24 \mathrm{~h}$ exposure period.

It is noteworthy that besides being induced via transcriptional activation, iNOS expression is also negatively regulated through increased iNOS protein degradation. Both pathways appear to be under independent control of different intercellular signaling factors. Thus, in RAW 264.7 cells IFN $\gamma$-dependent iNOS induction is inhibited by the 
immunosuppressive cytokine TGF- $\beta 1$ through enhanced protein degradation [16]. Similarly, the metabolic hormone GLP-1 (glucagon-like peptide-1) stimulates iNOS protein degradation and attenuates iNOS protein induction in RINm5F beta-cells challenged with IL-1 $\beta[17,18]$. Both signaling factors, TGF- $\beta 1$ and GLP-1, inhibit iNOS protein expression without any effect on iNOS gene transcription induced by pro-inflammatory cytokines [16,17]. Moreover, GLP-1 suppresses also NOX2 activity and ROS production by beta-cells, which will confer additional protection of beta-cells against cytokine-induced death and dysfunction [19]. Although we cannot exclude the possibility that brusatol via some unknown mechanism suppresses the translation of iNOS mRNA, we hypothesize that the main mechanism of action for brusatol is to increase iNOS protein degradation.

On molecular level the stability of the iNOS protein is regulated by the highly selective ECS/SPSB subfamily of E3 ubiquitin ligases [20,21]. At present, iNOS is the single substrate identified for ECS/SPSB. As we mentioned above, this ubiquitin system was suggested to be a target of brusatol, and its activation increases degradation of the Nrf2 protein by a mechanism which is poorly understood to date $[3,14,15]$. Nevertheless, as brusatol seems to cause similar effects on the stabilities of iNOS and Nrf2, we suggest that both effects are based on brusatoldependent alterations of the lifetimes of these proteins. In the future it will be interesting to verify this suggestion and examine possible common elements in signaling pathways implicated in the beta-cell response to brusatol, and those of GLP-1 and TGF- $\beta 1$.

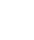

In summary, our findings demonstrate that brusatol completely blocks the sensitivity of beta-cells to pro-inflammatory cytokines and that it does so via a novel mechanism that involves post-transcriptional mechanisms. 
EndoC- $\beta \mathrm{H} 1$ cells were kindly provided by Raphael Scharfmann and Philippe Ravassard,

Paris, France. The present study was supported in part by the Swedish Diabetes Association and the family Ernfors Fund. The authors declare that there are no conflicts of interest.

\section{References}

[1] E. Mata-Greenwood, M. Cuendet, D. Sher, D. Gustin, W. Stock, J.M. Pezzuto, Brusatolmediated induction of leukemic cell differentiation and G(1) arrest is associated with downregulation of c-myc, Leukemia 16 (2002) 2275-2284.

[2] M. Cuendet, J.J. Gills, J.M. Pezzuto, Brusatol-induced HL-60 cell differentiation involves enhances the efficacy of chemotherapy by inhibiting the Nrf2-mediated defense mechanism, Proc. Natl. Acad. Sci. U.S.A. 108 (2011) 1433-1438. Sci. 1281 (2013) 16-35. 
[6] P. Pirot, A.K. Cardozo, D.L. Eizirik, Mediators and mechanisms of pancreatic beta-cell death in type 1 diabetes, Arq. Bras. Endocrinol. Metabol. 52 (2008) 156-165.

[7] D. Mokhtari, A. Al-Amin, K. Turpaev, T. Li, O. Idevall-Hagren, J. Li, A. Wuttke, R.G. Fred, P. Ravassard, R. Scharfmann, A. Tengholm, N. Welsh, Imatinib mesilate-induced phosphatidylinositol 3-kinase signalling and improved survival in insulin-producing cells: role of Src homology 2-containing inositol 5'-phosphatase interaction with c-Abl, Diabetologia 56 (2013) 1327-1338.

[8] P. Ravassard, Y. Hazhouz, S. Pechberty, E. Bricout-Neveu, M. Armanet, P. Czernichow, R. Scharfmann, A genetically engineered human pancreatic $\beta$ cell line exhibiting glucose-inducible insulin secretion, J. Clin. Invest. 121 (2011) 3589-3597.

[9] E. Eruslanov, S. Kusmartsev, Identification of ROS using oxidized DCFDA and flowcytometry, Methods Mol. Biol. 594 (2010) 57-72.

[10] J.R. Weaver, T.R. Holman, Y. Imai, A. Jadhav, V. Kenyon, D.J. Maloney, J.L. Nadler, G. Rai, A. Simeonov, D.A. Taylor-Fishwick, Integration of pro-inflammatory cytokines, 12lipoxygenase and NOX-1 in pancreatic islet beta cell dysfunction, Mol. Cell. Endocrinol. 358 (2012) 88-95.

[11] A.M. Mohammed, K. Syeda, T. Hadden, A. Kowluru, Upregulation of phagocyte-like NADPH oxidase by cytokines in pancreatic beta-cells: attenuation of oxidative and nitrosative stress by 2-bromopalmitate, Biochem. Pharmacol. 85 (2013) 109-114.

[12] S. Lenzen, J. Drinkgern, M. Tiedge, Low antioxidant enzyme gene expression in pancreatic islets compared with various other mouse tissues, Free Radic. Biol. Med. 20 (1996) 463-466.

[13] W. Tang, J. Xie, S. Xu, H. Lv, M. Lin, S. Yuan, J. Bai, Q. Hou, S. Yu, Novel nitric oxidereleasing derivatives of brusatol as anti-inflammatory agents: design, synthesis, biological evaluation, and nitric oxide release studies, J. Med. Chem. 57 (2014) 7600-7612. 
[14] X.J. Chao, Z.W. Chen, A.M. Liu, X.X. He, S.G. Wang, Y.T. Wang, P.Q. Liu, C.

315 Ramassamy, S.H. Mak, W. Cui, A.N. Kong, Z.L. Yu, Y.F. Han, R.B. Pi, Effect of tacrine-3-

caffeic acid, a novel multifunctional anti-Alzheimer's dimer, against oxidative-stress-induced cell death in HT22 hippocampal neurons: involvement of Nrf2/HO-1 pathway, CNS Neurosci. Ther. 20 (2014) 840-850.

[15] A. Olayanju, I.M. Copple, H.K. Bryan, G.T. Edge, R.L. Sison, M.W. Wong, Z.Q. Lai, Z.X. Lin, K. Dunn, C.M. Sanderson, A.F. Alghanem, M.J. Cross, E.C. Ellis, M. Ingelman-Sundberg, H.Z. Malik, N.R. Kitteringham, C.E. Goldring, B.K. Park, Brusatol provokes a rapid and transient inhibition of Nrf2 signaling and sensitizes mammalian cells to chemical toxicityimplications for therapeutic targeting of Nrf2, Free Radic. Biol. Med. 78 (2015) 202-212.

[16] T. Mitani, M. Terashima, H. Yoshimura, Y. Nariai, Y. Tanigawa, TGF-beta1 enhances degradation of IFN-gamma-induced iNOS protein via proteasomes in RAW 264.7 cells, Nitric Oxide 13 (2005) 78-87. Kim, Exendin-4 inhibits interleukin-1beta-induced iNOS expression at the protein level, but not at the transcriptional and posttranscriptional levels, in RINm5F beta-cells, J. Endocrinol. 202 (2009) 65-75.

[18] D. Rondas, M. Bugliani, W. D'Hertog, K. Lage, M. Masini, E. Waelkens, P. Marchetti, C.

Mathieu, L. Overbergh, Glucagon-like peptide-1 protects human islets against cytokine-mediated $\beta$-cell dysfunction and death: a proteomic study of the pathways involved, J. Proteome Res. 12 (2013) 4193-4206.

[19] N. Li, B. Li, T. Brun, C. Deffert-Delbouille, Z. Mahiout, Y. Daali, X.J. Ma, K.H. Krause, P. Maechler, NADPH oxidase NOX2 defines a new antagonistic role for reactive oxygen species and cAMP/PKA in the regulation of insulin secretion, Diabetes 61 (2012) 2842-2850.

[20] T. Nishiya, K. Matsumoto, S. Maekawa, E. Kajita, T. Horinouchi, M. Fujimuro, K.

Ogasawara, T. Uehara, S. Miwa, Regulation of inducible nitric-oxide synthase by the SPRY 
[21] K. Matsumoto, T. Nishiya, S. Maekawa, T. Horinouchi, K. Ogasawara, T. Uehara, S. Miwa, The ECS(SPSB) E3 ubiquitin ligase is the master regulator of the lifetime of inducible nitricoxide synthase, BBRC 409 (2011) 46-51.

\section{Legends to figures}

Fig. 1. Brusatol protects against cytokine-induced beta-cell death. $\beta$ TC6 (A), RIN-5AH (B), and human EndoC- $\beta \mathrm{H} 1(\mathrm{C})$ cells were treated or not with brusatol $(50 \mathrm{nM})$ for $30 \mathrm{~min}$ prior to simultaneous exposure to IL-1 $\beta$ and IFN $\gamma$ for additional $24 \mathrm{~h}$. Cells were labeled with fluorescent dye PI, trypsinized and analyzed by flow cytometry. Each bar represents the mean \pm SD of four paired independent experiments. $p<0.0001(*), p<0.001(* *)$ versus cytokines. NT, not treated cells.

Fig. 2. Dose-dependence of brusatol-mediated protection of beta-cells from pro-inflammatory cytokines. $\beta$ TC6 were treated with brusatol at the indicated concentrations for 30 min prior to simultaneous exposure to IL-1 $\beta$ and IFN $\gamma$ for an additional $24 \mathrm{~h}$. Cells were labeled with fluorescent dye PI, trypsinized and analyzed by flow cytometry. Each bar represents the mean \pm SD of four paired independent experiments. $p<0.0001(*), p<0.001(* *)$ versus cytokines.

Fig. 3. Brusatol inhibits cytokine-induced ROS production in $\beta$ TC6 cells. (A) Representative histogram of intracellular ROS levels measured by flow cytometry. (B) Quantification of data above. The oxidative sensitive dye DCFDA was used for the measurement of ROS production. Geometric mean (Geo Mean) was used to calculate the total intensity of fluorescence. Each bar 
represents the mean $\pm \mathrm{SD}$ of four paired independent experiments. $\beta$ TC6 cells were treated or not with $50 \mathrm{nM}$ brusatol for $30 \mathrm{~min}$ prior to simultaneous exposure to IL-1 $\beta$ and IFN $\gamma$ for $11 \mathrm{~h}$ followed by incubation in fresh serum-free RPMI medium at the presence of DCFDA for 45 min and subsequent incubation in fresh serum-containing RPMI medium for additional $1 \mathrm{~h}$. Cells were trypsinized and analyzed by flow cytometry. $p<0.05\left(^{*}\right)$ versus cytokines; $p<0.005\left(^{\#}\right.$ ) versus control.

Fig. 4. Brusatol inhibits activation of iNOS protein expression in $\beta$ TC6 cells exposed to IL-1 $\beta$ without significant effect on iNOS mRNA induction and NF- $\kappa$ B nuclear accumulation. (A) Western blot image of iNOS expression in $\beta$ TC6 cells exposed to IL-1 $\beta$ and brusatol. Cell culture was pretreated with brusatol $(50 \mathrm{nM})$ for $30 \mathrm{~min}$ and further incubated in the simultaneous presence of IL-1 $\beta$ for additional $8 \mathrm{~h}$. Whole cell lysates were analyzed by immunoblotting with iNOS antibodies. Antibodies to $\beta$-actin were used as a loading control. (B, left) Quantification of data above. iNOS protein level in untreated $\beta$ TC6 cells was assigned a value of 100. Each bar represents the mean $\pm \mathrm{SD}$ of at least three independent experiments. (B, right) The expression of iNOS genes in $\beta$ TC6 cells exposed to IL-1 $\beta$ and brusatol. Cells were pretreated with brusatol $(50 \mathrm{nM})$ for $30 \mathrm{~min}$, further incubated in the simultaneous presence of IL-1 $\beta$ for additional $4 \mathrm{~h}$, and analyzed by RT-PCR. iNOS mRNA level was normalized to the level of GAPDH mRNA. The basal content of iNOS mRNA in control cells was assigned a value of 1. Results represent the mean of three independent experiments ( \pm SD). (C) Brusatol did not

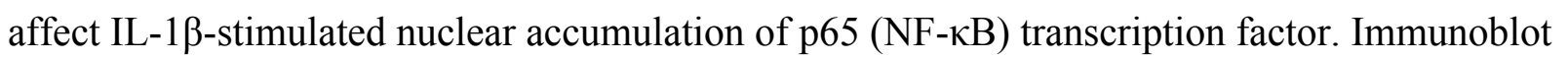
analysis of nuclear extracts from $\beta$ TC6 cells incubated with IL-1 $\beta$ for $40 \mathrm{~min}$ at the presence or absence of $50 \mathrm{nM}$ brusatol. Antibodies to PTBP2 and ERK 1/2 were used for control of loading and nuclear purification. Representative results of three independent experiments are shown (panels A and D). $p<0.005(*)$ versus IL-1 $\beta$ (panel B). 
391 
$392 \quad$ Figure 1

393

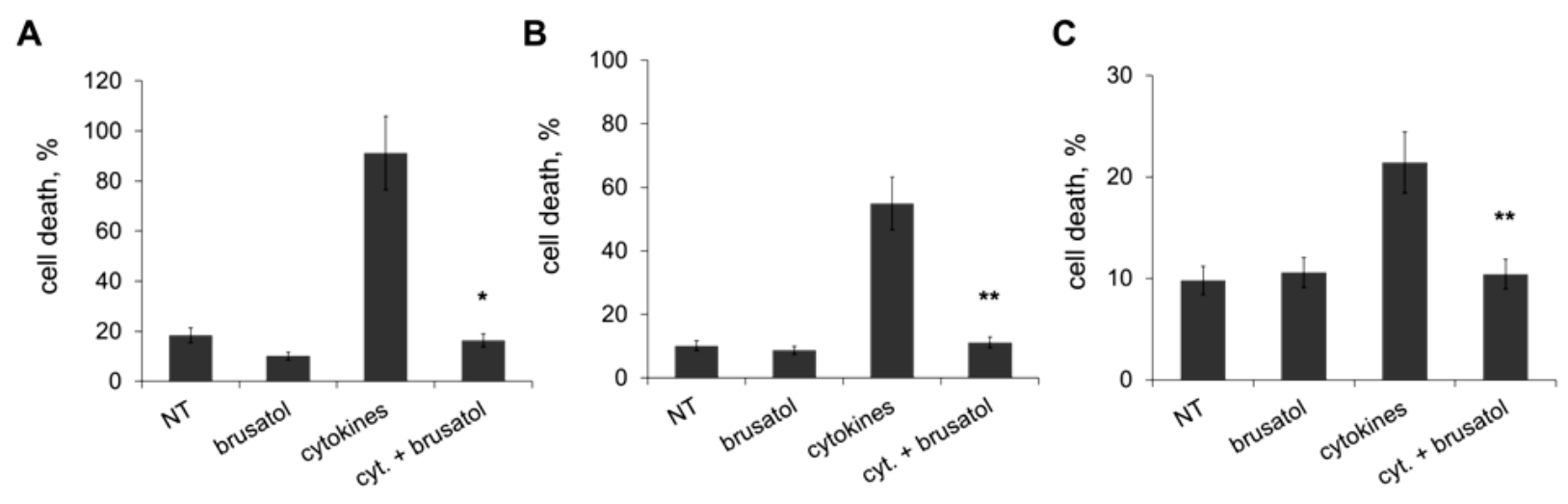

394

395 
A

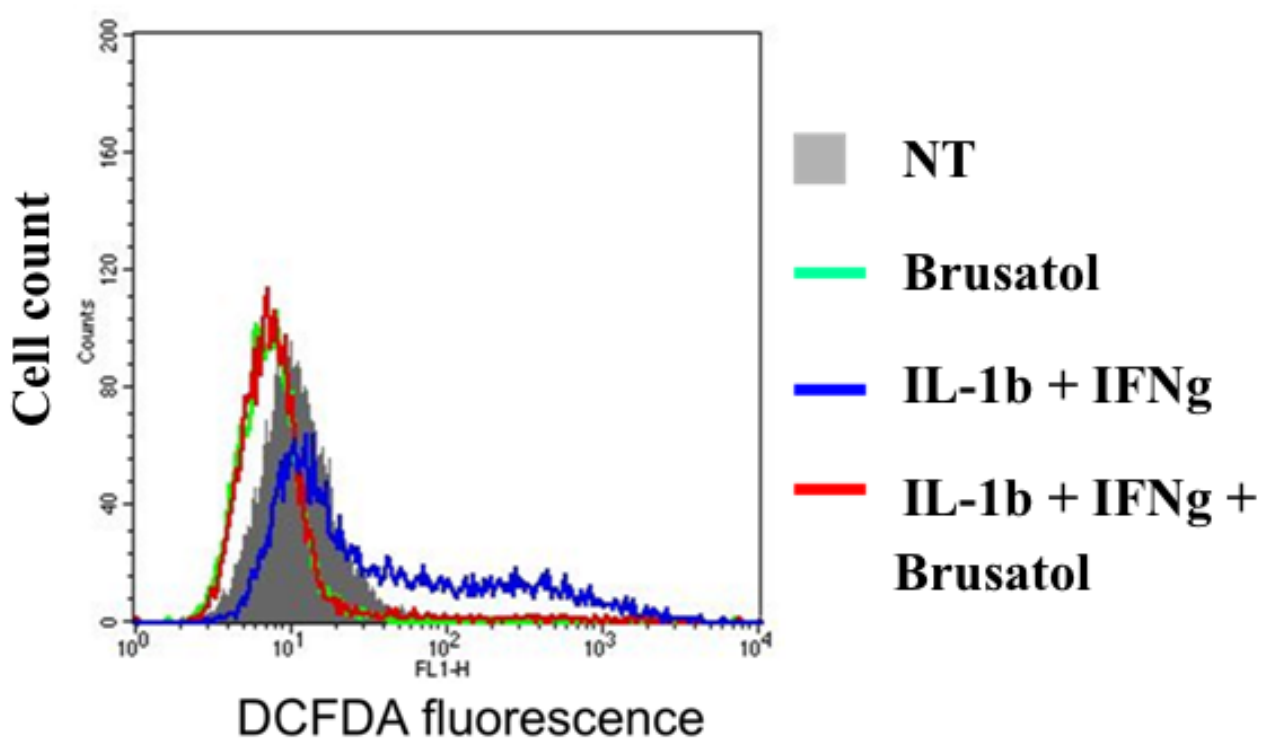

B

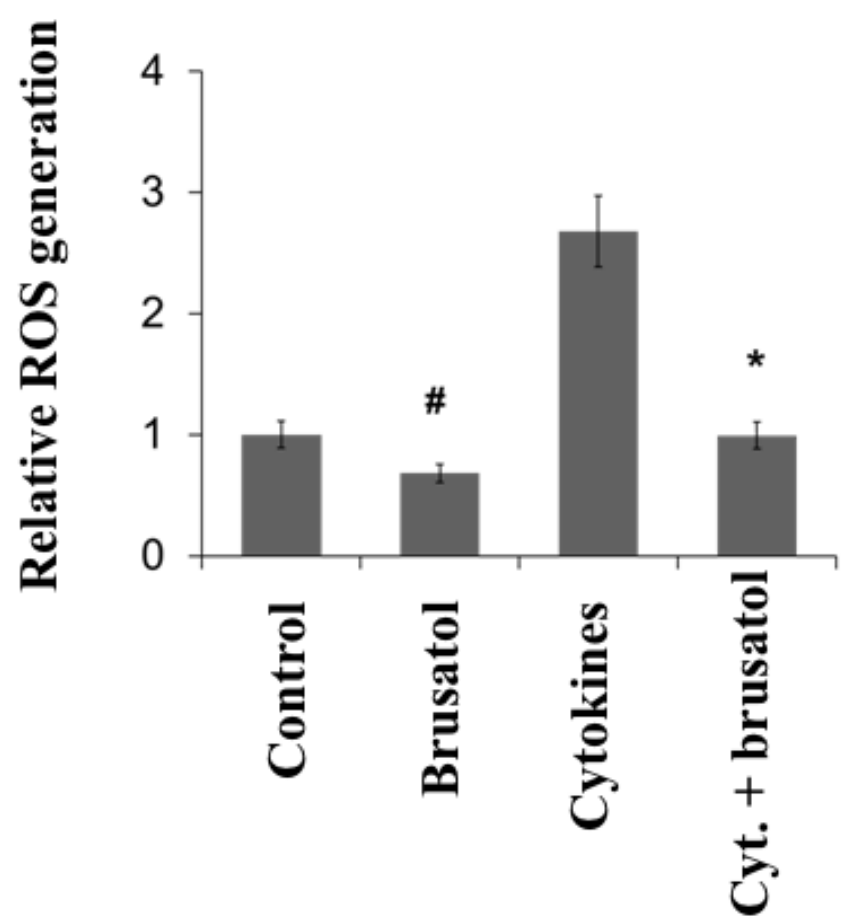


Figure 3

A

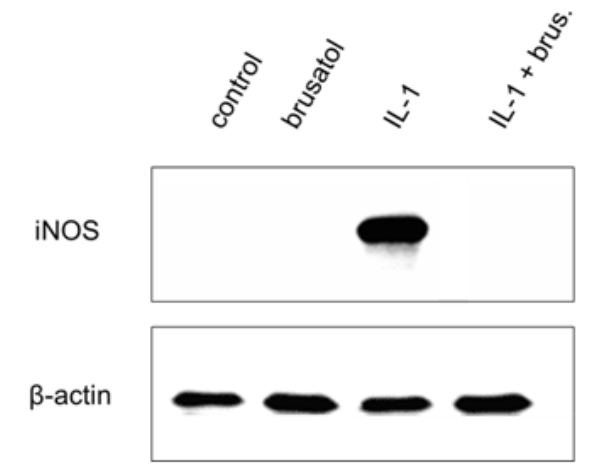

C

B
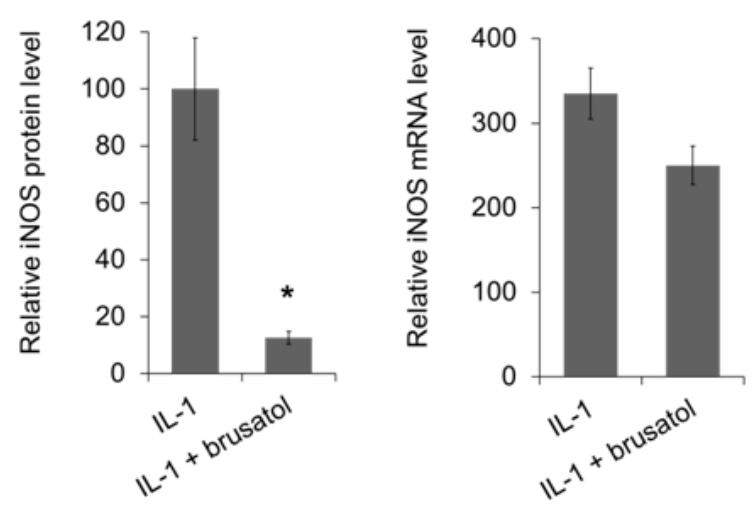

PTB-2

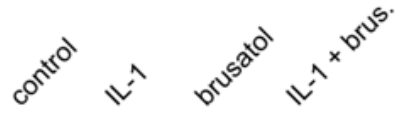

p65 /NF-kB

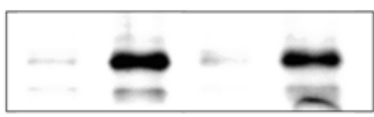

ERK

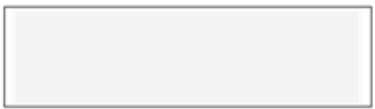

400

401 